\title{
Multiple Reaction Fronts in the Oxidation-Reduction of Iron-Rich Uranium Ores
}

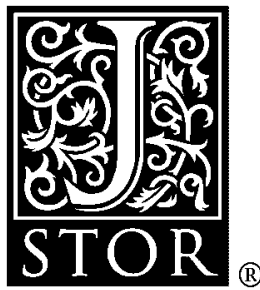

\author{
J. N. Dewynne; A. C. Fowler; P. S. Hagan
}

SIAM Journal on Applied Mathematics, Vol. 53, No. 4. (Aug., 1993), pp. 971-989.

Stable URL:

http://links.jstor.org/sici?sici=0036-1399\%28199308\%2953\%3A4\%3C971\%3AMRFITO\%3E2.0.CO\%3B2-E

SIAM Journal on Applied Mathematics is currently published by Society for Industrial and Applied Mathematics.

Your use of the JSTOR archive indicates your acceptance of JSTOR's Terms and Conditions of Use, available at

http://www.jstor.org/about/terms.html. JSTOR's Terms and Conditions of Use provides, in part, that unless you have obtained prior permission, you may not download an entire issue of a journal or multiple copies of articles, and you may use content in the JSTOR archive only for your personal, non-commercial use.

Please contact the publisher regarding any further use of this work. Publisher contact information may be obtained at http://www.jstor.org/journals/siam.html.

Each copy of any part of a JSTOR transmission must contain the same copyright notice that appears on the screen or printed page of such transmission.

The JSTOR Archive is a trusted digital repository providing for long-term preservation and access to leading academic journals and scholarly literature from around the world. The Archive is supported by libraries, scholarly societies, publishers, and foundations. It is an initiative of JSTOR, a not-for-profit organization with a mission to help the scholarly community take advantage of advances in technology. For more information regarding JSTOR, please contact support@jstor.org. 


\title{
MULTIPLE REACTION FRONTS IN THE OXIDATION-REDUCTION OF IRON-RICH URANIUM ORES*
}

\author{
J. N. DEWYNNE†, A. C. FOWLER $\ddagger$, AND P. S. HAGAN§
}

\begin{abstract}
This paper describes the oxidation of iron-rich uranium-bearing rocks by infiltration of groundwater. A reaction-diffusion model is set up to describe the sequence of reactions involving iron oxidation, uranium oxidation and reduction, sulphuric acid production, and dissolution of the host rock that occur. On a geological timescale of millions of years, the reactions occur very fast in very thin reaction fronts. It is shown that the redox front that separates oxidized (orange) rock from reduced (black) rock must actually consist of two separate fronts that move together, at which the two separate processes of uranium oxidation and iron reduction occur, respectively. Between these fronts, a high concentration of uranium is predicted. The mechanics of this process are not specific to uranium-mediated redox reactions, but apply generally and may be used to explain the formation of concentrated ore deposits in extended veins. On the long timescales of relevance, a quasi-static response results, and the problem can be solved explicitly in one dimension. This provides a framework for studying more realistic two-dimensional problems in fissured rocks and also for the future study of uraninite nodule formation.
\end{abstract}

Key words. reaction-diffusion, oxidation-reduction, reaction fronts

AMS(MOS) subject classifications. $35 \mathrm{~K} 57,76 \mathrm{~S} 05,86 \mathrm{~A} 60$

1. Introduction. When a container of radioactive waste is buried underground, it eventually corrodes, and leakage of radioactive material to the surrounding rock occurs. Depending on the chemistry of the rock, many different reactions may occur. A particular case concerns the oxidation and reduction of uranium ores by infiltrating groundwater, since $\mathrm{UO}_{3}$ is relatively soluble (and hence potentially transportable to the water supply), whereas $\mathrm{UO}_{2}$ is essentially insoluble. It is therefore of concern to those involved with radioactive waste disposal to understand the mechanics of uranium transport through reduction and oxidation reactions.

The same phenomena more or less take place in naturally occurring uranium ores, and a graphic example is provided in an open cast uranium mine, such as the Osamu Utsumi mine at Poços de Caldas in Brazil (see Fig. 1). Here, the rock is iron-rich, containing 2 percent by weight of pyrite $\left(\mathrm{FeS}_{2}\right)$, which acts as a reducing agent. As oxygenated surface water penetrates the rock, the pyrite is oxidized, as is evidenced by the brilliant orange colour of the (rusty) oxidized rock. The front between oxidized and reduced rock is extremely sharp and owes its irregular shape (Fig. 1) to the presence of fissures, where the groundwater penetrates preferentially. The mine at Poços de Caldas provides the basis for an international study, which is described by Smellie et al. [8]. One purpose of the project is to "produce a model of geochemical transport across redox fronts, with special attention to the understanding of long-term, large-scale movements of redox-sensitive natural series radionuclides."

The host rock contains reduced uranium $\left(\mathrm{UO}_{2}\right.$, or uranium in the $\mathrm{U}^{4+}$ state). As the water infiltrates, this reduced uranium is oxidized, becomes soluble, and is thus transported toward the redox front, where it is reduced and precipitates once more. An interesting phenomenon is that uraninite nodules are observed to occur ahead of the redox front; these are small $(\sim 1 \mathrm{~cm})$ spherical regions in which the concentration of $\mathrm{UO}_{2}$ is extremely high.

* Received by the editors May 6, 1991, accepted for publication (in revised form) July 16, 1992.

$\dagger$ Faculty of Mathematical Studies, University of Southampton, Highfield, Southampton SO9 5NH, England.

¥ Mathematical Institute, Oxford University, Oxford OX13LB, England.

$\S$ M.S. B265, C-3, Los Alamos National Laboratory, Los Alamos, New Mexico 87545. 


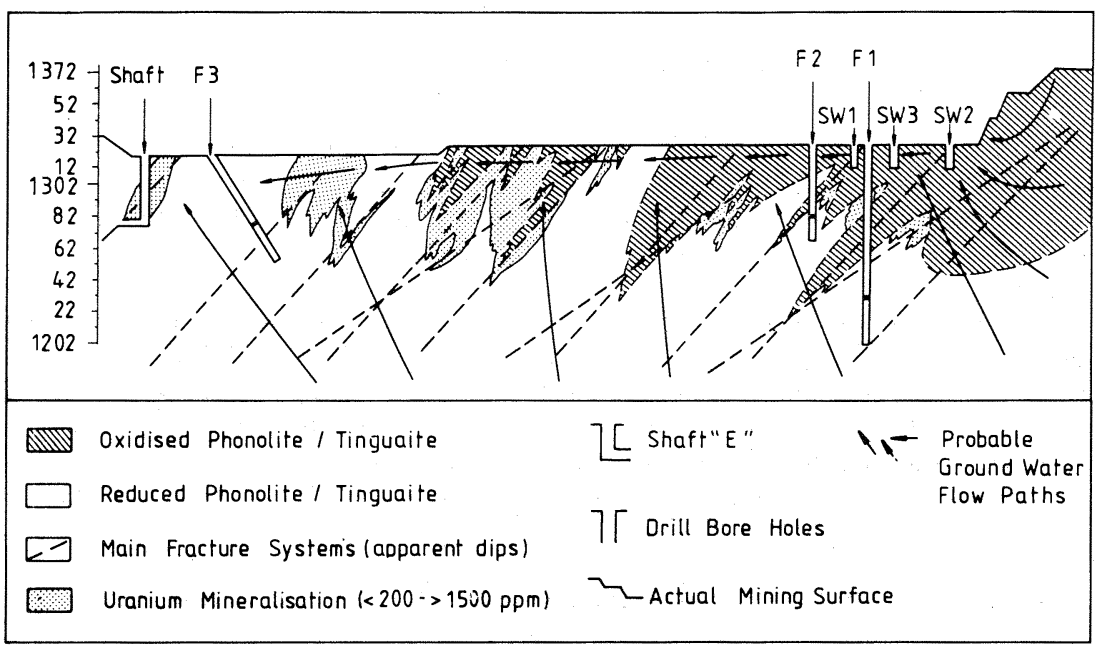

FIG. 1. A sketch of a cross section of the Osamu Utsumi mine (after [8]). The shaded regions represent oxidized rock and tend to be centred on the main fracture systems.

There are three problems of immediate practical interest, both scientifically and for the containment problem. The first is to determine the rate at which the redox front will advance. This can most easily be studied using a uniform one-dimensional model, that is, where we ignore fissures. It is, in fact, necessary to study this one-dimensional problem to provide a computationally efficient model for the case when fissures are present (which is the second problem and which will be reserved for future work). The third problem is the mechanism of nodule formation and propagation. Again, this requires a onedimensional description in which a model for the formation mechanism can be addressed. This too will form the subject for a future study.

In this paper, we therefore address only the case where a redox front advances uniformly. The novel feature that we find is that the redox front, in fact, consists of two separate fronts, where two separate reactions occur. The fronts are tied together because the supply of reactants at each front is determined by the reaction at its neighboring front. We find the timescales are so long that the whole system evolves quasi-statically and can be explicitly solved. This provides a basis for the further studies referred to above.

Economic geologists have done a good deal of work in this area, and complicated reaction-diffusion models have been posed and solved numerically (e.g., [10]). The formation of ore deposits in sandstones (called roll front deposits) as a result of dissolution and precipitation kinetics is well understood and is comparable to our description of the redox front, in which oxidation and reduction occur at separate fronts (that delineate the boundaries of the ore deposit).

In a sequence of papers, Ortoleva and colleagues [4]-[6] have analyzed reactiondiffusion models relevant to redox front propagation in porous rock because of infiltration of oxygenated groundwater. They are particularly interested in various kinds of spatial instabilities, such as fingering or banding, and draw attention to a mechanism for these, which they dub the "reaction-infiltration" instability. This involves the feedback between precipitation, porosity, and flow rate. In geological environments, such as those under consideration here, they draw attention to two crucial asymptotic limits: one is that of 
fast reaction rates, which enables the system to be reduced to a set of "slow" reactiondiffusion equations separated by moving reaction "fronts"; the second is "solid density asymptotic," exemplified by the solubility of a mineral in pore water being much less than its gross density. The effect is that the transport timescales are fast, and, as a result, we can solve quasi-static equations with the time dependence exhibited by the moving fronts.

In realistic modeling of the chemical reactions (for example, Walsh et al. [10] solve a model with 26 minerals and 60 aqueous species), these approximations have also been introduced. Most notably, equilibrium chemistry is assumed [10], [1]. This corresponds to the assumption of fast reaction rates and is very accurate for the long timescales of relevance here. When the concentration of aqueous oxidant species is much less than the concentration of the reducing minerals present in the rock, then solid density asymptotics are relevant [3], in which the advection and diffusion of the concentrations adjust to steady conditions much more rapidly than reaction front movement occurs. These approximations enable us to solve the one-dimensional problem virtually explicitly, and we find that the redox front moves (after an initial transient) at a constant rate, an idea that is also well known (e.g., [2], [4]).

Recently, Cross et al. [1] used a computer model combining detailed chemical equilibria equations with advective and diffusive transport to model the redox front propagation at Poços de Caldas. This paper has a similar purpose, but our approach is orthogonal. We have written the simplest possible description of the chemistry involved. While we thus lose important details of the reactive processes, we find a significant gain insofar as we are able to explicitly describe the front propagation process. In particular, we give explicit formulae for the front propagation speed and for the thickness of the ore-bearing redox zone (i.e., the region between the oxidation and reduction fronts). An important inference is that the front speed is determined solely by conservation of the total number of oxidant atoms, entering as dissolved $\mathrm{O}_{2}$. The implication is that twodimensional models may be easily adaptable to analysis by using parameterizations based on the present results. A second important inference is that straightforward numerical models almost certainly predict the uraninite $\mathrm{U}^{4+}$ concentration incorrectly, since the redox zone thickness may well be much smaller than any realistic grid spacing (cf. [1, Fig. 3]). We consider that a coherent future modeling strategy will marry the ideas of the present work with more realistic model chemistry.

We wish to emphasize the distinguishing features of the present model, which go beyond the fast reaction rate, "solid density" asymptotics of Ortoleva et al. [6]. The presence of two uranium species mediates the redox reaction and actually splits the front into two parts. As far as we are aware, such an idea is completely novel. Moreover, the analysis of the split redox front is facilitated by a third approximation, that $\delta$ is small (where $\delta$ is the ratio of solubilities of $\mathrm{U}^{4+}$ and $\mathrm{U}^{6+}$ ). As a consequence of this analysis, we are impelled to consider nonequilibrium precipitation-dissolution kinetics, again in a way that appears novel and goes beyond the precipitation modeling of Sultan et al. [9]. In fact, we find that, if $\kappa$ is a measure of the precipitation-dissolution rate, then the singular limits $\kappa \rightarrow \infty, \delta \rightarrow 0$ do not commute, and it is then essential to retain the nonequilibrium kinetics for a realistic answer.

2. Mathematical model. We base our model on the conditions observed in the Poços de Caldas mine [1]. The reduced rock has a porosity of about 4 percent, contains about 2 percent by weight of pyrite, and has a density of $2.2 \times 10^{3} \mathrm{~kg} \mathrm{~m}^{-3}$. The oxidized rock has a porosity of 20 percent. This implies that about $300 \mathrm{~kg} \mathrm{~m}^{-3}$ of oxidized rock is dissolved and washed away. This occurs because the redox reactions produce sulphuric 
acid that is mainly responsible for the dissolution. Specifically, potassium feldspar reacts to form clay minerals and silica, some of which is dissolved. The infiltrating groundwater is, in fact, quite acidic, because of dissolved $\mathrm{CO}_{2}$. Most of this acid is expended hydrolyzing the oxidized rock, and a slowly moving hydrolysis front is found between the surface and the redox front. The hydrolyzed rock near the surface is found to be relatively soft and subject to significant erosion. The $\mathrm{pH}$ of the groundwater changes from about 3.03.6 in the hydrolyzed region, to about 5.5-6.2 in the reduced/oxidized regions [1]. For our purposes, however, the only effect of the hydrolysis front is to increase the $\mathrm{pH}$ of the infiltrating groundwater, and we ignore the hydrolysis front in what follows. The flow rate of water from the surface is about $10^{-1} \mathrm{~m}^{3}$ per square meter per year, and contains oxygen at a concentration of $10^{-2} \mathrm{~kg} \mathrm{~m}^{-3}\left(\mathrm{H}_{2} \mathrm{O}\right)$. In the undisturbed rock, the uranium oxide concentration is $6.3 \times 10^{-2} \mathrm{~kg} \mathrm{~m}^{-3}$ (rock). The solubility limits of the uranium oxides are (approximately) $10^{-2} \mathrm{~kg} \mathrm{~m}^{-3}\left(\mathrm{H}_{2} \mathrm{O}\right)$ in the oxidized region and $10^{-6} \mathrm{~kg} \mathrm{~m}^{-3}$ in the reduced region. That is, $\mathrm{UO}_{2}\left(\right.$ or $\mathrm{U}^{4+}$ ) is slightly soluble in water; it is more soluble in sulphuric acid, and thus it is realistic to suppose that the solubility of $\mathrm{U}^{4+}$ depends on the acid concentration.

Our present aim is to model the redox front. For the eventual purposes of studying nodule formation, it is necessary to include a description of how the acid produced at the redox front reacts with feldspar in the rock. A list of principal chemical constituents that must be included in the model is $\mathrm{FeS}_{2}$ (pyrite), $\mathrm{O}_{2}$ (oxygen), $\mathrm{H}^{+}$(acid), $\mathrm{U}^{4+}$ (reduced uranium $\mathrm{UO}_{2}$ ), $\mathrm{U}^{6+}$ (oxidized uranium $\mathrm{UO}_{3}$ ), and $\mathrm{S}$ ( silicates and other minerals present in the primitive rock that are soluble in acid). The uranium is present in both aqueous $\mathrm{U}_{a q}$, and precipitate $\mathrm{U}_{s}$ form. These species undergo reactions as follows:

$$
\begin{aligned}
& \text { (i) } 4 \mathrm{FeS}_{2}+15 \mathrm{O}_{2} \rightarrow 16 \mathrm{H}^{+}\left[+8 \mathrm{SO}_{4}^{2-}\right] \text { (slow), } \\
& \text { (ii) } 2 \mathrm{U}^{4+}+\mathrm{O}_{2} \rightarrow 2 \mathrm{U}^{6+}, \\
& \text { (iii) } 15 \mathrm{U}^{6+}+2 \mathrm{FeS}_{2} \rightarrow 15 \mathrm{U}^{4+}+8 \mathrm{H}^{+} \quad \text { (slow), } \\
& \text { (iv) } \mathrm{U}_{s}^{4+} \rightleftharpoons \mathrm{U}_{a q}^{4+}, \\
& \text { (v) } \mathrm{U}_{s}^{6+} \rightleftharpoons \mathrm{U}_{a q}^{6+}, \\
& \text { (vi) } m^{\prime} \mathrm{S}+\mathrm{H}^{+} \rightarrow \text { dissolved salts, clay }
\end{aligned}
$$

These reactions represent, respectively,

(i) the overall redox reaction,

(ii) oxidation of uranium,

(iii) reduction of uranium,

(iv) dissolution/precipitation of $\mathrm{U}^{4+}$,

(v) dissolution/precipitation of $\mathrm{U}^{6+}$,

(vi) silicate reaction.

Note that the combined effect of reactions (ii) and (iii) is to produce reaction (i). We have only included the constituents in these reactions that are necessary to determine the model. While all the reactions are fast on a geological timescale, the surface-mediated ones are relatively slow, as only the surface atoms of the solid are available as reaction sites; these are marked slow in (2.1). We suppose that the oxidation reaction (ii) occurs exclusively in the aqueous solution.

Let $u, v, w, x, r, c, h$, and $s$ denote the variable concentrations as follows:

$$
\begin{array}{ll}
u=\left[\mathrm{U}_{s}^{4+}\right], & v=\left[\mathrm{U}_{a q}^{4+}\right], \quad w=\left[\mathrm{U}_{a q}^{6+}\right], \quad x=\left[\mathrm{U}_{s}^{6+}\right], \\
r=\left[\mathrm{FeS}_{2}\right], & c=\left[\mathrm{O}_{2}\right], \quad h=\left[\mathrm{H}^{+}\right], \quad s=M[\mathrm{~S}] .
\end{array}
$$

These are molar concentrations, measured in units of $\mathrm{mol} \mathrm{m}^{-3}$ ( of rock for $u, r$; of water for $v, w, c, h)$. Because of the variety of species that can be leached from the ore, it is 
more convenient to measure $s$ in units of $\mathrm{kg} \mathrm{m}^{-3}$ (of rock). As a consequence, the molar silicate concentration has been multiplied by "its" molecular weight $M$. The constant $m^{\prime}$ is a representative stoichiometric coefficient. Typical values for the Poços de Caldas mine are

$$
u \sim 0.265\left(6.3 \times 10^{-2} \mathrm{~kg} \mathrm{~m}^{-3}\right), \quad v \sim 4.2 \times 10^{-6}\left(10^{-6} \mathrm{~kg} \mathrm{~m}^{-3}\right),
$$

$$
\begin{gathered}
w \sim 0.042\left(10^{-2} \mathrm{~kg} \mathrm{~m}^{-3}\right), \quad r \sim 350\left(42 \mathrm{~kg} \mathrm{~m}^{-3}\right), \quad c \sim 0.313\left(10^{-2} \mathrm{~kg} \mathrm{~m}^{-3}\right), \\
s \sim 300 \mathrm{~kg} \mathrm{~m}^{-3}, \quad h \sim 0.3\left(3 \times 10^{-4} \mathrm{~kg} \mathrm{~m}^{-3}\right) .
\end{gathered}
$$

The values of $v, w$, and $c$ are inferred from likely solubility limits, and $h$ from its oxygen equivalent; $r$ and $u$ are the initial values in the rock; $s$ is the quantity of feldspar that is dissolved. Note that $r, s \gg u, w, c, h \gg v$; the size of $x$ is as yet unknown. We denote the porosity by $\phi$; thus $\phi \sim 1 / 5$ in the oxidized region, while $\phi=1 / 25$ in the reduced rock. In fact, $\phi$ is also a variable with $\phi=\phi(s) ; \phi$ may also depend on $u$ and $x$, and this may be important in later studies of nodule formation. Let $z$ denote the coordinate perpendicular to the earth's surface, pointing downward. We denote by $J$ the (constant) vertical flux of water, taken to be $J \sim 10^{-1} \mathrm{~m}^{3}$ (water) $\mathrm{m}^{-2}$ (rock) $y^{-1}$. The diffusive flux of species $y$ is $-\phi(D / \tau) \nabla y$, where $D$ is the diffusion coefficient, and $\tau$ is the tortuosity. In the absence of information, we take $\tau=1$; we take $D \sim 3 \times 10^{-2} \mathrm{~m}^{2} \mathrm{y}^{-1}$ for all species (except $u, x, r$, and $s$ that are nonaqueous, and neither diffuse nor are advected).

Let the molar rates of reaction of the steps (i) to (vi) of (2.1) be $r_{1}, r_{2}, r_{3}, r_{4}$ and $r_{-4}, r_{5}$ and $r_{-5}$, and $r_{6}$, respectively ( $r_{4}$ being the rate of the forward reaction in $(2.1)$ (iv), $r_{-4}$ being the rate of the backward reaction, and similarly for $r_{5}$ and $r_{-5}$ in $\left.(2.1)(\mathrm{v})\right)$. Then, the equations governing the reaction scheme $(2.1)$ may be written as

$$
\begin{gathered}
(\phi c)_{t}+(J c)_{z}=\left(\phi D c_{z}\right)_{z}-15 r_{1}-r_{2}, \\
u_{t}=-r_{4}+r_{-4}, \\
(\phi v)_{t}+(J v)_{z}=\left(\phi D v_{z}\right)_{z}+r_{4}-r_{-4}-2 r_{2}+15 r_{3}, \\
(\phi w)_{t}+(J w)_{z}=\left(\phi D w_{z}\right)_{z}+2 r_{2}-15 r_{3}+r_{5}-r_{-5}, \\
x_{t}=-r_{5}+r_{-5}, \\
r_{t}=-4 r_{1}-2 r_{3}, \\
(\phi h)_{t}+(J h)_{z}=\left(\phi D h_{z}\right)_{z}+16 r_{1}+8 r_{3}-r_{6}, \\
s_{t}=-m r_{6},
\end{gathered}
$$

where $m=m^{\prime} M$ and has units of $\mathrm{kg} \mathrm{mol}^{-1}$. The reaction rates $r_{1}(r, c), r_{2}(v, c)$, and $r_{3}(w, r)$ can generally be expected to be complicated nonlinear functions of the concentrations, satisfying $r_{i}(0, y)=r_{i}(y, 0)=0$. Their precise form is not relevant, and it is helpful to suppose that $r_{1} \propto r c, r_{2} \propto v c$, and $r_{3} \propto w r$. The rates $r_{4}, r_{-4}$ and $r_{5}, r_{-5}$ are related to the solubilities of $\mathrm{U}^{4+}$ and $\mathrm{U}^{6+}$ and may depend on $h$.

The diffusing species $c, v, w$, and $h$ require boundary conditions. We take these to be

$$
c=c_{0}, \quad\left[J-\phi D \frac{\partial}{\partial z}\right](v, w, h)=0 \quad \text { at } z=0
$$

and

$$
u \rightarrow u_{0}, \quad c, w, h \rightarrow 0 \quad \text { as } z \rightarrow \infty .
$$

We apply a boundary condition for $u$ rather than $v$ since the primitive rock contains a concentration $u_{0}$ of $\mathrm{U}_{s}^{4+}$. The value of $v$ is tied to that of $u$ by solubility (see below). 
We now exploit the idea that the rate constants are mainly extremely large (the possible exceptions being $r_{1}, r_{3}, r_{6}$ ) on the geological timescales of interest. The resultant "fast reaction rate" asymptotics have been expounded by Ortoleva et al. [6]. This implies that the irreversible reactions take place over extremely thin transition layers in which diffusion is balanced with the reactions. Outside these transition layers, the rates of the irreversible reactions decay to zero exponentially. It is a feature of these types of asymptotic problems, where the decay outside the transition layers is exponential, that in an appropriate asymptotic expansion, the reaction rates vanish to all algebraic orders ( of the small parameters, namely the $\left.1 / r_{i}\right)$. It follows that the leading-order behavior of the reactants is then actually described by the advective diffusion equations with the reaction rates set to zero. (A comparable example occurs in the boundary layer theory of Rayleigh-Bénard convection at high Rayleigh number $R_{a}$, where the interior (outer solution ) temperature is isothermal to all orders of the small parameter $R_{a}^{-1 / 3}$ [7].) Although we allow $r_{1}, r_{3}$, $r_{6}$ to be relatively small, in fact we also put $r_{1}$ and $r_{3}$ to zero outside reaction fronts as we expect the oxidation reactions to be rate limited by the aqueous species, and thus very fast in practice. We do not necessarily assume that $r_{6}$ is fast, however. This applies to the irreversible equations, excepting those containing the dissolution reactions (iv) and $(\mathrm{v})$, since the corresponding reactions do not occur in a thin layer, and the equilibrium is not transcendentally accurate. To obtain flux equations from $(2.4 \mathrm{~b})-(2.4 \mathrm{e})$ we must therefore add these equations to eliminate the $r_{ \pm 4}$ and $r_{ \pm 5}$ terms (see [6, p. 1001] for an explanation of this). We can then equate the reaction terms identically to zero outside any transition layer, yielding

$$
u_{t}+(\phi v)_{t}+(J v)_{z}=\left(\phi D v_{z}\right)_{z}, \quad x_{t}+(\phi w)_{t}+(J w)_{z}=\left(\phi D w_{z}\right)_{z} .
$$

The other equations, relating $u$ to $v$ and $x$ to $w$ outside transition layers, come from $(2.4 \mathrm{~b})$ and $(2.4 \mathrm{e})$

$$
\begin{aligned}
& u_{t}=-r_{4}+r_{-4}, \\
& x_{t}=-r_{5}+r_{-5} .
\end{aligned}
$$

Now, equating the other reaction rates to zero shows that $r=0$ or $c=w=0$ (from (2.4), and since all concentrations are nonnegative). If $r=0$, then (2.4a) implies $c v=0$; thus, in either event, we have $c v=r w=0$ everywhere outside transition layers. We assume that the solubility limit of $v$ is given by a function of $u$ and $h$, as shown in Fig. 2 . Specifically, we assume that in equlibrium

$$
\begin{aligned}
& u=0, \quad v<v_{0} f(h), \\
& v=v_{0} f(h), \quad u>0,
\end{aligned}
$$

where the dimensionless $O(1)$ function $f(h)$ is shown in Fig. 2; we expect $v_{0}$ to be small (that is, $v_{0} \ll u$ ). In fact, we will assume that $x$ is in equilibrium, that is, $r_{5}=-r_{-5}$ in ( $2.9 \mathrm{~b}$ ), but we do not assume equilibrium for $u$. We model the precipitation dissolution terms in $(2.8 \mathrm{a})$ as follows:

$$
u_{t}= \begin{cases}k(v-f) u, & v<f, \\ k(v-f), & v \geqq f .\end{cases}
$$

The second of these is similar to the precipitation model of Sultan et al. [9]. In an earlier work [4], a linear adsorption-desorption was used. We consider (2.10) more realistic. 


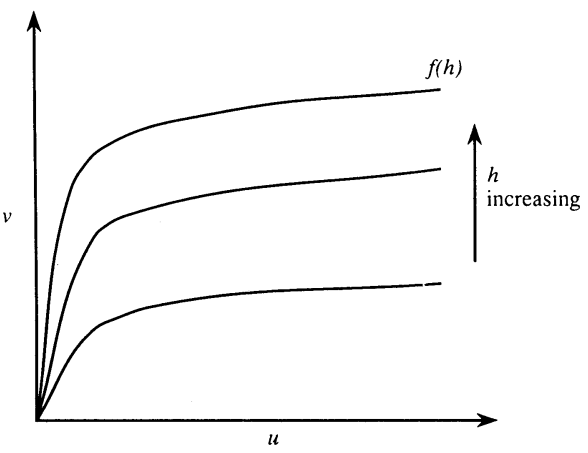

(a)

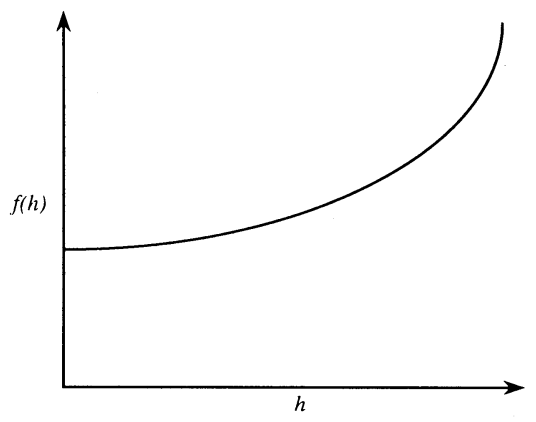

(b)

FIG. 2. (a) Solubility of aqueous $\mathrm{U}^{4+}$ as a function of precipitate $\mathrm{U}^{4+}$, for various levels of acid concentration; (b) Variation of solubility limit with acid concentration.

Substituting (2.9) into (2.7) and appending the other equilibrium forms of (2.4), we have the advective diffusion equations

$$
\begin{aligned}
(\phi c)_{t}+(J c)_{z} & =\left(\phi D c_{z}\right)_{z}, \\
(u+\phi v)_{t}+(J v)_{z} & =\left(\phi D v_{z}\right)_{z}, \\
(x+\phi w)_{t}+(J w)_{z} & =\left(\phi D w_{z}\right)_{z}, \\
(\phi h)_{t}+(J h)_{z} & =\left(\phi D h_{z}\right)_{z}-r_{6}, \\
s_{t} & =-m r_{6} .
\end{aligned}
$$

The equation for $r, \partial r / \partial t=0$, shows that $r$ is piecewise constant ( that is, constant between reaction layers). While it is likely that the redox reactions are fast on a geological timescale, we infer that feldspar dissolution is slower, since the formation of uraninite nodules suggests that acid can permeate ahead of the redox front to some extent, and this can only be the case if $r_{6}$ is small.

These equations (2.11) apply between any reaction fronts that exist. Across such fronts, jump conditions apply that are determined by exact conservation laws. Examining (2.4), we find three such conservation laws, in the form

$$
\begin{gathered}
(4 r+\phi h-s / m)_{t}+(J h)_{z}=\left(\phi D h_{z}\right)_{z}, \\
(u+\phi v+x+\phi w)_{t}+(J(v+w))_{z}=\left(\phi D\left(v_{z}+w_{z}\right)\right)_{z}, \\
(4 \phi c+2 \phi w-15 r+2 x)_{t}+(J(4 c+2 w))_{z}=\left(\phi D\left(4 c_{z}+2 w_{z}\right)\right)_{z} .
\end{gathered}
$$

From these, we deduce the three jump conditions, below, at a reaction front moving with speed $V$ :

$$
\begin{aligned}
{[4 r+\phi h-s / m]_{-}^{+} V } & =\left[J h-\phi D h_{z}\right]_{-}^{+}, \\
{[u+\phi v+x+\phi w]_{-}^{+} V } & =\left[J(v+w)-\phi D\left(v_{z}+w_{z}\right)\right]_{-}^{+}, \\
{[4 \phi c+2 \phi w-15 r+2 x]_{-}^{+} V } & =\left[J(4 c+2 w)-\phi D\left(4 c_{z}+2 w_{z}\right)\right]_{-}^{+},
\end{aligned}
$$

where [ $]_{-}^{+}$represents the jump across the front. In addition, matching the diffusing species profiles in a reaction layer to an outer solution with finite (and, when scaled, 
$O(1)$ ) gradients can only be achieved if, at leading order (in $1 / r_{i}$ ), the diffusing species are continuous across reaction fronts; thus

$$
[v]_{-}^{+}=[c]_{-}^{+}=[w]_{-}^{+}=[h]_{-}^{+}=0 .
$$

In addition, the assumption that the silicate reaction is slow implies that

$$
[s]_{-}^{+}=0 .
$$

Thus, (2.13) simplifies to

$$
\begin{aligned}
4[r]_{-}^{+} V & =\left[-\phi D h_{z}\right]_{-}^{+}, \\
{[u+x]_{-}^{+} V } & =\left[-\phi D\left(v_{z}+w_{z}\right)\right]_{-}^{+}, \\
{[-15 r+2 x]_{-}^{+} V } & =\left[-\phi D\left(4 c_{z}+2 w_{z}\right)\right]_{-}^{+},
\end{aligned}
$$

where we have used the continuity of water flux $[J]_{-}^{+}=0$ and that $[\phi]_{-}^{+}=0$ if $\phi=\phi(s)$.

This completes our derivation of the advection-diffusion equations. One additional, very accurate approximation, can now be made. The advective timescale is $t \sim z / J$, while the diffusive timescale is $t \sim z^{2} / D$. Taking $z \sim 10 \mathrm{~m}, J \sim 10^{-1} \mathrm{~m} \mathrm{y}^{-1}, D \sim$ $3 \times 10^{-2} \mathrm{~m}^{2} \mathrm{y}^{-1}$, these are, respectively, about 100 years and $10^{4}$ years. This suggests that, after an initial transient, advection dominates diffusion, and, furthermore, if $J \gg$ $V$, then the fronts evolve quasi-statically. Since, because of the large amount of $\mathrm{FeS}_{2}$ and the small amount of $\mathrm{O}_{2}$, this will be the case, we can anticipate that the whole problem may be treated using a quasi-static approximation (that is, we neglect the time partial derivatives in the equations).

3. The basic redox front. We first illustrate the solution by considering the uncontaminated case, where uranium is entirely absent, $u=v=w=x=0$, and the redox reaction ( $i)$, together with the etching reaction ( vi ), are the only processes. This situation has been considered by Ortoleva et al. [4], for example, and we repeat their results here for ease of reference in the subsequent analysis.

In the quasi-static approximation, we must solve

$$
(J c)_{z}=\left(\phi D c_{z}\right)_{z}, \quad(J h)_{z}=\left(\phi D h_{z}\right)_{z}-r_{6},
$$

with the jump conditions at the front

$$
\begin{gathered}
{[c]_{-}^{+}=[h]_{-}^{+}=0,} \\
4[r]_{-}^{+} V=\left[-\phi D h_{z}\right]_{-}^{+}, \\
-15[r]_{-}^{+} V=\left[-4 \phi D c_{z}\right]_{-}^{+} .
\end{gathered}
$$

In addition, reaction equilibrium implies (from (2.4)) that

$$
r_{1}=0 \Rightarrow c r=0 \text {. }
$$

We therefore expect a redox front to exist, behind which $r=0$ and in front of which $c=0$, as shown in Fig. 3. The acid produced at the redox front dissolves the rock. The model is completed by specifying a relationship between $\phi$ and $s$. Most simply,

$$
\phi=\phi_{0}+b\left(s_{0}-s\right),
$$

where the data from the Poços de Caldas mine suggests that

$$
\phi_{0}=0.04, \quad b=5.33 \times 10^{-4} \mathrm{~m}^{3} \mathrm{~kg}^{-1}
$$




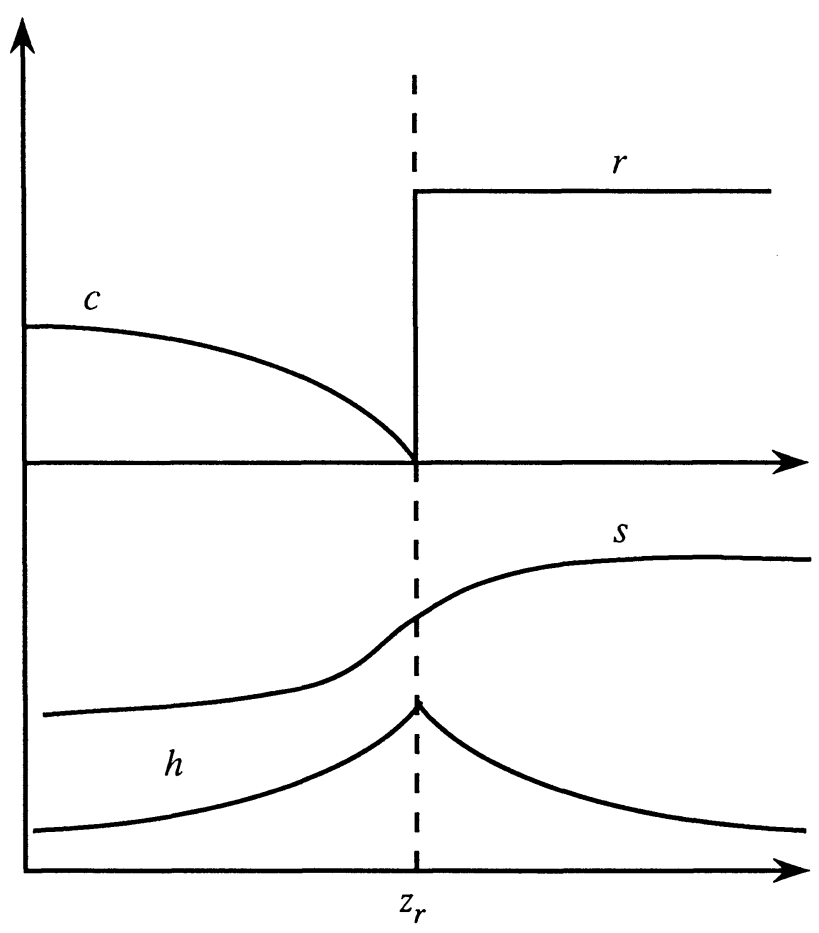

FIG. 3. Redox front propagation in the absence of uranium. Shown are profiles of $c, r, h$, and $s$.

(so that $\phi=0.2$ when $\Delta s=s_{0}-s$ corresponds to $300 \mathrm{~kg} \mathrm{~m}^{-3}$ ). In this first illustrative example, however, we take $\phi$ to be constant.

We denote the redox front by $z_{r}(t)$. Suppose that $r=r_{0}$ in $z>z_{r}(t)$; solving for $c$ in $z<z_{r}(t)$, we find that

$$
c=\frac{c_{0}\left[\exp \left(J z_{r} / \phi D\right)-\exp (J z / \phi D)\right]}{\left[\exp \left(J z_{r} / \phi D\right)-1\right]},
$$

where, at $z=z_{r}(t)$,

$$
-15 r_{0} V=4 \phi D c_{z},
$$

and where $V=d z_{r} / d t$. We thus find that

$$
V=\frac{4 J\left(c_{0} / r_{0}\right)}{15\left[1-\exp \left(-z_{r} / l\right)\right]},
$$

where

$$
l=\phi D / J
$$

Using $\phi=0.2, D=3 \times 10^{-2} \mathrm{~m}^{2} \mathrm{y}^{-1}, c_{0}=0.313 \mathrm{M} \mathrm{m}^{-3}$, and $r_{0}=350 \mathrm{M} \mathrm{m}^{-3}$, we have (3.10) $l \sim 6 \mathrm{~cm}, \quad V / J \sim 4 c_{0} / 15 r_{0} \sim 0.24 \times 10^{-3}, \quad V \sim 0.24 \times 10^{-4} \mathrm{~m} \mathrm{y}^{-1}$.

The quasi-static approximation is certainly valid for $z_{r} \gtrsim l$. For $z_{r} \ll l$, (3.8) implies that $z_{r} \sim\left[J l\left(c_{0} / r_{0}\right) t\right]^{1 / 2}$, and the neglect of the time partial derivative is only valid if $t \gg l\left(c_{0} / r_{0}\right) / J$, that is, if $z_{r} \gg l\left(c_{0} / r_{0}\right)$. In practice, $l\left(c_{0} / r_{0}\right)$ is so small that the details 
of the initial transient are irrelevant. For relevant values of $z_{r} \gg l$, we have the asymptotic steady-state velocity

$$
V \sim 4 J c_{0} / 15 r_{0}
$$

The front takes $4 \times 10^{5}$ years to travel 10 meters (this figure is consistent with the computer model predictions of [1]). To complete the solution, we determine the acid profile. (In other redox reactions, if acid is not produced, then the above description is complete.) We suppose that reaction of feldspar in the rock occurs at a rate proportional to acid concentration; thus

$$
r_{5}=k h \text {. }
$$

Solving for $h$ and assuming that $z_{r} \gg l$, we obtain, approximately,

$$
h=\left\{\begin{array}{lc}
A \exp \left[m^{+}\left(z-z_{r}\right)\right], & z<z_{r}, \\
A \exp \left[m^{-}\left(z-z_{r}\right)\right], & z>z_{r},
\end{array}\right.
$$

where

$$
m^{ \pm}=\frac{1}{2 \phi D}\left[J \pm \sqrt{J^{2}+4 k \phi D}\right]
$$

and the jump condition (3.2b) applied at $z_{r}$ gives

$$
4 r_{0} V=-\phi D\left[h_{z}\right]_{-}^{+},
$$

whence

$$
A=\frac{4 r_{0} V}{\phi D\left(m^{+}-m^{-}\right)}=\frac{4 r_{0} V}{\sqrt{J^{2}+4 k \phi D}} .
$$

We can now calculate the feldspar concentration. For $z \gg l$, we have $\partial / \partial t \sim$ $V \partial / \partial z$, and $s$ satisfies

$$
V s_{z}=-m r_{5}=-m k h,
$$

with $s \rightarrow s_{0}$ as $z \rightarrow \infty$. We find that

$$
s=s_{r}+\frac{m k A}{m^{ \pm} V}\left[1-\exp \left(m^{ \pm}\left(z-z_{r}\right)\right)\right]
$$

for $z \gtrless z_{r}$, where the value of $s$ at $z_{r}$, $s_{r}$ is given by

$$
s_{r}=s_{0}-\frac{m k A}{m^{+} V} .
$$

It follows that the total consumption of feldspar by the redox front $\Delta s=s_{0}-s(-\infty)$ is given by

$$
\Delta s=\frac{m k A}{V}\left(\frac{1}{m^{+}}-\frac{1}{m^{-}}\right)=m r_{0} .
$$

Since $r_{0}=350 \mathrm{M} \mathrm{m}^{-3}$ and $\Delta s=300 \mathrm{~kg} \mathrm{~m}^{-3}$ we infer that $m$ should take the value 0.214 $\mathrm{kg} \mathrm{mol}^{-1}$. For example, potassium feldspar $\mathrm{KAlSi}_{3} \mathrm{O}_{8}$ has a molecular weight of 278 , consistent with a stoichiometric coefficient $m^{\prime}$ of 0.77 (as opposed to a value of one given by Cross et al. [1]). We are, of course, blurring the distinction between reaction (to form clay minerals) and dissolution (that can remove material from the rock). 
4. Uranium catalyzed oxidation-reduction fronts. We now return to the full problem, where the uranium reactions (ii), (iii), (iv), and (v) are included. It transpires that the oxidation reaction (ii) and the reduction reaction (iii), which, on combination, are equivalent to the redox reaction (i), actually replace it. The redox reaction (i) itself does not occur, and the uranium facilitates the reaction by splitting the single front into two fronts. At the rear, oxidation of uranium occurs (ii); this produces $\mathrm{U}^{6+}$, which is then transported downward to the reduction front, where reaction (iii) reduces the pyrite. The acid produced by this reaction dissolves feldspars, as indicated in the previous section. The scheme is represented in Fig. 4. It will turn out that the oxidation front $z_{O}$ and the reduction front $z_{R}$ are close together because of the small solubility of $\mathrm{U}^{4+}$ in acidic water. Following the analysis of $\S 3$, it will be convenient to nondimensionalize the system. If $z_{O}$ is close to $z_{R}$, we can expect the length scale $\left(z_{R}-z_{O}\right)$ to be such that diffusion of $\mathrm{U}_{a q}^{4+}, v$, balances advection of $\mathrm{U}_{a q}^{4+}$; otherwise, $\mathrm{U}^{4+}$ cannot be effectively transported backward. We therefore define

$$
z=z_{O}(t)+l z^{*}
$$

where $l$ is determined by (3.9), where we take $\phi=\phi_{0}$, the porosity in the reduced region. The relevant timescale is then $l / V$, and, from $\S 3$, we expect $\dot{z}_{O}=V \sim J\left(c_{0} / r_{0}\right)$; thus

$$
t=\frac{l r_{0}}{J c_{0}} t^{*}, \quad V=J\left(c_{0} / r_{0}\right) V^{*}
$$

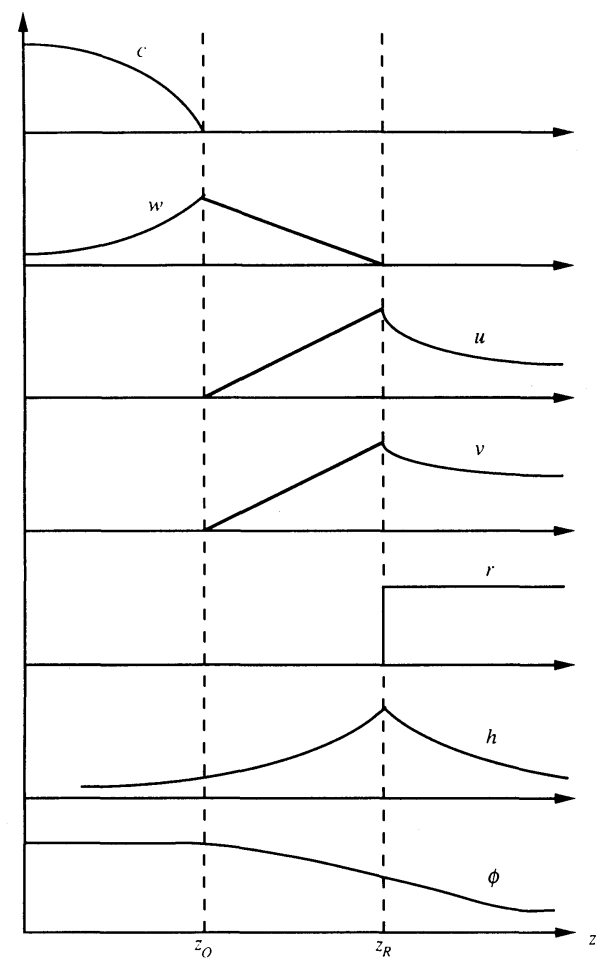

FIG. 4. Redox front propagation with uranium present. Two separate fronts exist, with a solubility front between them. 
Also, we put

$$
\begin{aligned}
& c=c_{0} c^{*}, \quad h=c_{0} h^{*}, \quad r=r_{0} r^{*}, \quad u=c_{0} u^{*}, \quad v=v_{0} v^{*}, \\
& w=v_{0} w^{*}, \quad x=\phi_{0} v_{0} x^{*}, \quad s=s_{0}-(\Delta s) s^{*}, \quad \phi=\phi_{0} \phi^{*},
\end{aligned}
$$

where $c_{0}=0.313 \mathrm{M} \mathrm{m}^{-3}, r_{0}=350 \mathrm{M} \mathrm{m}^{-3}, u_{0}=0.265 \mathrm{M} \mathrm{m}^{-3}, \phi_{0}=0.04, v_{0}=4.2 \times$ $10^{-6} \mathrm{M} \mathrm{m}^{-3}$, and $w_{0}=0.042 \mathrm{M} \mathrm{m}^{-3}$. We substitute these into (2.9) and (2.10), boundary conditions $(2.5),(2.6)$, and jump conditions (2.13) and (2.14); in addition, reaction equilibrium requires from (2.4) that $r_{1}=r_{2}=r_{3}=0$, whence

$$
c r=r w=c v=0 \text {, }
$$

everywhere (except in the reaction fronts).

We obtain the equations (dropping the asterisks)

$$
\begin{gathered}
\varepsilon\left[(\phi c)_{t}-V(\phi c)_{z}\right]+c_{z}=\left(\phi c_{z}\right)_{z}, \\
\varepsilon\left(\frac{\partial}{\partial t}-V \frac{\partial}{\partial z}\right)[u+\delta \phi v]+\delta v_{z}=\left(\delta v_{z}\right)_{z}, \\
\varepsilon\left[(x+\phi w)_{t}-V(x+\phi w)_{z}\right]+w_{z}=\left(\phi w_{z}\right)_{z}, \\
\varepsilon\left[(\phi h)_{t}-V(\phi h)_{z}\right]+h_{z}=\left(\phi h_{z}\right)_{z}-\lambda h, \\
\phi=1+\beta s, \\
\phi_{t}-V \phi_{z}=\mu h,
\end{gathered}
$$

$$
u_{t}-V u_{z}=\left\{\begin{array} { l l } 
{ \kappa ( v - f ( h ) ) u } & { \text { if } v < f ( h ) , } \\
{ \kappa ( v - f ( h ) ) } & { \text { if } v > f ( h ) , }
\end{array} \quad \left\{\begin{array}{ll}
x=0 & \text { if } w<1 / \nu \\
x>0 & \text { if } w=1 / \nu
\end{array}\right.\right.
$$

where

$$
\begin{aligned}
& \beta=b(\Delta s) / \phi_{0}, \quad \delta=\phi_{0} v_{0} / c_{0}, \quad \varepsilon=\phi_{0} c_{0} / r_{0}, \quad \lambda=k l / J, \\
& \mu=b m k h_{0}[t] / \phi_{0}, \quad \nu=v_{0} / w_{0}, \quad \kappa=k v_{0}[t],
\end{aligned}
$$

and $[t]$ is the timescale $[t]=l r_{0} / J c_{0}$. Using previous estimates, we have

$$
[t] \sim 700 \mathrm{y}, \quad \beta \sim 4, \quad \delta \sim 0.6 \times 10^{-6}, \quad \varepsilon \sim 3.6 \times 10^{-5}, \quad \nu \sim 10^{-4} ;
$$

$\lambda, \mu$, and $\kappa$ are undetermined at present. Note that $\varepsilon \gg \delta$ in (4.5b).

The relevant boundary conditions for these equations are, from (2.5) and (2.6),

$$
\begin{gathered}
c \rightarrow 1, \quad\left(1-\phi \frac{\partial}{\partial z}\right)\{v, w, h\} \rightarrow 0 \quad \text { as } z \rightarrow-\infty, \\
u \rightarrow u_{\alpha}=u_{0} / c_{0}, \quad c, w, h \rightarrow 0, \quad v \rightarrow f\left(0^{+}\right) \quad \text { as } z \rightarrow+\infty .
\end{gathered}
$$

Note that the profiles in Fig. 4 satisfy the equilibrium constraints (4.4), and, by choice of origin $\left(z_{O}\right)$, we have

$$
c=0, \quad u=0 \quad \text { on } z=0 .
$$

Finally, we have the jump conditions (2.13); that is, the $v, c, w$, and $h$ are continuous at reaction fronts, and the flux conditions (2.15) become (dimensionlessly)

$$
\begin{aligned}
4[r]_{-}^{+} V_{i} & =-\left[\phi h_{z}\right]_{-}^{+}, \\
E[u+N x]_{-}^{+} V_{i} & =\left[-\phi\left(w_{z}+v_{z}\right)\right]_{-}^{+}, \\
{[-15 r+2 p \varepsilon x]_{-}^{+} V_{i} } & =\left[-\phi\left(4 c_{z}+2 \delta^{\prime} w_{z}\right)\right]_{-}^{+},
\end{aligned}
$$


where $V_{i}$ is the velocity of the relevant front $(i=O$ or $i=R)$. The parameters are defined by

$$
\begin{aligned}
& E=\varepsilon / \delta=c_{0}^{2} / v_{0} r_{0} \sim 60, \\
& \delta^{\prime}=\nu p=v_{0} / c_{0}=\delta / \phi_{0} \sim 10^{-5}, \\
& N=\phi_{0} w_{0} / c_{0} \sim 0.6 \times 10^{-2}, \\
& p=w_{0} / c_{0} \sim 0.13 .
\end{aligned}
$$

Providing that $w<1 / \nu$ in the solutions, then $\mathrm{U}^{6+}$ never reaches its solubility limit, and, in that case, we can assume that $x=0$ everywhere. The validity of this assumption is checked below.

As $\varepsilon, \delta \ll 1$, it is appropriate to seek a leading-order approximation by letting $\varepsilon$, $\delta \rightarrow 0$, but retaining $E$ as an $O(1)$ parameter for the moment. At leading order, we have

$$
\begin{aligned}
c_{z} & =\left(\phi c_{z}\right)_{z}, \\
w_{z} & =\left(\phi w_{z}\right)_{z}, \\
h_{z} & =\left(\phi h_{z}\right)_{z}-\lambda h, \\
\phi_{t}-V \phi_{z} & =\mu h, \\
E\left(u_{t}-V u_{z}\right)+v_{z} & =\left(\phi v_{z}\right)_{z}, \\
u_{t}-V u_{z} & = \begin{cases}\kappa(v-f) u, & v<f, \\
\kappa(v-f), & v>f,\end{cases}
\end{aligned}
$$

with boundary conditions

$$
\begin{gathered}
c \rightarrow 1,\left(1-\phi \frac{\partial}{\partial z}\right)\{v, w, h\} \rightarrow 0 \quad \text { as } z \rightarrow-\infty, \\
c=0, \quad u=0 \quad \text { on } z=0, \\
u \rightarrow u_{\infty}, \quad c, w, h \rightarrow 0, \quad v \rightarrow f\left(0^{+}\right) \quad \text { as } z \rightarrow+\infty,
\end{gathered}
$$

and jump conditions given by $(4.1)$.

For large time, we expect the solutions to be quasi-steady; we also suppose that $\mu$, $\lambda \sim O(1)$. Although nonlinear, the problem may be simplified by defining the modified space variable

$$
\zeta=\int_{0}^{z} \frac{d z}{\phi}
$$

whence

$$
\begin{gathered}
c_{\zeta}=c_{\zeta \zeta}, \\
-E V u_{\zeta}+v_{\zeta}=v_{\zeta \zeta}, \\
w_{\zeta}=w_{\zeta \zeta}, \\
-V u_{\zeta}= \begin{cases}\kappa \phi(v-f) u, & v<f, \\
\kappa \phi(v-f), & v>f,\end{cases}
\end{gathered}
$$

and

$$
h_{\zeta}=h_{\zeta \zeta}-\lambda \phi h, \quad-V \phi_{\zeta}=\mu \phi h,
$$


with the jump conditions (4.11) being

$$
\begin{gathered}
4[r]_{-}^{+} V_{i}=-\left[h_{\zeta}\right]_{-}^{+}, \\
E[u]_{-}^{+} V_{i}=-\left[v_{\zeta}+w_{\zeta}\right]_{-}^{+}, \\
15[r]_{-}^{+} V_{i}=\left[4 c_{\zeta}+2 \delta^{\prime} w_{\zeta}\right]_{-}^{+} .
\end{gathered}
$$

We retain the $\delta^{\prime}$ in $(4.18 \mathrm{c})$ to obtain a nondegenerate solution. We denote the position of the reduction front by $\zeta=\rho(t)$. We find that

$$
\begin{gathered}
c=1-e^{\zeta}, \quad \zeta<0, \\
w=A e^{\zeta}, \quad \zeta<0, \\
w=w_{0}+B e^{\zeta}, \quad \zeta>0,
\end{gathered}
$$

where, to satisfy $(4.18)$, we have

$$
w_{0}=2 / \delta^{\prime}, \quad B=-2 e^{-\rho} / \delta^{\prime}, \quad A=B+2 / \delta^{\prime} .
$$

To satisfy the jump condition $\left(4.18 \mathrm{c}\right.$ ) at $\zeta=\rho$, we require (as $V_{i}=V+\dot{\rho}$ there) that

$$
V+\dot{\rho}=4 / 15 \text {. }
$$

Thus the two-front solution collapses to the solution given in $\S 3$ as $\rho \rightarrow 0$.

It is natural to illustrate the nature of the solutions by making two further simplifications. If the precipitation time $1 /\left(k v_{0}\right)$ is small compared to the timescale $[t] \sim 700$ years, then $\kappa$ is large, and we might expect that equilibrium (2.9a) will apply. Furthermore, it is attractive to suppose that $f$ is constant; i.e., the uranium solubility is independent of the acid concentration. We find, however, that if we make both of these assumptions, then $v_{\zeta}=0$ when $u>0$, and this implies (from (4.16c)) that $u_{\zeta}=0$, and hence $u=u_{\infty}$ in $\zeta>\rho$. There is then no mechanism by which uranium collected from $\zeta<0$ can be transported forward, and it all resides between the oxidation and reduction fronts. This leads to absurdly high concentrations of uranium between these fronts, since, in addition, we find that $\rho \sim \delta^{\prime} \ll 1$ in this case. Such concentrations are not seen in practice, and this suggests that the simplifications above cannot be realistic. We examine, in turn, the effect of nonequilibrium precipitation kinetics and $\mathrm{H}^{+}$solubility dependence on the solutions of our model.

4.1. Nonequilibrium precipitation. First, we suppose that $\kappa$ in $(4.16)$ is finite and that $f=1$. We expect that in $0<\zeta<\rho$ there is a solubility front at $z=z_{S}$ or $\zeta=\sigma(t)$, where $v=f$, and we anticipate that $v<f$ for $\zeta<\sigma$ and $v>f$ for $\zeta>\sigma$. At this front, $h_{\zeta}$ and $w_{\zeta}$ are continuous (so that (4.19c) applies for $0<\zeta<\rho$ and (4.21) is valid); in addition, the finite rate of precipitation implies that $h$ will be continuous, which, in turn, requires that $v_{\zeta}$ be continuous at $\sigma$. The equations satisfied by $u$ and $v$ are then

$$
\begin{aligned}
-V u_{\zeta} & = \begin{cases}\kappa \phi(v-1) u, & \zeta<\sigma, \\
\kappa \phi(v-1), & \zeta>\sigma,\end{cases} \\
-E V u_{\zeta} & =v_{\zeta \zeta}-v_{\zeta} .
\end{aligned}
$$

For ease of exposition, we assume that $\phi \sim 1$, as is the case if $\mu$ is small. Then

$$
v_{\zeta \zeta}-v_{\zeta}=\kappa E(v-1), \quad \zeta>\sigma
$$

and

$$
-E V u=v_{\zeta}-v+\text { constant. }
$$


The equation for $v$ is nonlinear, but we can simplify it by making use of the anticipated result that $\rho$ is small. It then follows that $v_{\zeta} \sim$ constant on the interval $(0, \sigma)$, and, from (4.18b), using (4.19) and (4.20), we have

$$
v_{\zeta} \sim 2 / \delta^{\prime}, \quad v \sim 2 \zeta / \delta^{\prime}, \quad 0<\zeta<\sigma
$$

(in fact, this is valid for $0<\zeta<\rho$ ), and thus

$$
\begin{array}{cl}
u=0, & \zeta<0, \\
u \sim 2 \zeta / \delta^{\prime} E V, & 0<\zeta<\sigma .
\end{array}
$$

Hence, from (4.23), we find that

$$
v=1+C \exp \left(-\frac{1}{2}(\sqrt{1+4 \kappa E}-1) \zeta\right)
$$

in $\zeta>\rho$, and continuity of $v$ requires that

$$
1+C \exp \left(-\frac{1}{2}(\sqrt{1+4 \kappa E}-1) \rho\right)=2 \rho / \delta^{\prime} .
$$

We anticipate that $\rho \sim \delta^{\prime}$, so that this is approximately

$$
C=2\left(\rho / \delta^{\prime}\right)-1 .
$$

Also, $v=1$ on $\zeta=\sigma$, so that, from (4.25),

$$
\sigma=\delta^{\prime} / 2 \text {. }
$$

At $\zeta=\rho,(4.18 \mathrm{~b})$ is automatically satisfied to leading order. We determine $\rho$ by continuity of $u$ at $\zeta=\rho$, since (4.24) implies that

$$
u=u_{\infty}+\frac{C}{2 E V}(\sqrt{1+4 \kappa E}+1) \exp (-(\sqrt{1+4 \kappa E}-1) \zeta / 2),
$$

whence approximately, using (4.29) and (4.26) at $\zeta=\rho$,

$$
u_{\infty}+\frac{1}{2 E V}\left(2\left(\rho / \delta^{\prime}\right)-1\right)(\sqrt{1+4 \kappa E}+1)=\frac{2}{E V}\left(\rho / \delta^{\prime}\right),
$$

and thus

$$
\rho \sim \delta^{\prime}\left(\frac{\sqrt{1+4 \kappa E}+1-2 E V u_{\infty}}{2(\sqrt{1+4 \kappa E}-1)}\right) .
$$

We see that $\rho \sim \delta^{\prime}$ as expected, and $u=O(1)$, with a decreasing exponential tail ahead of the redox front. As $\kappa$ becomes large (that is, rapid precipitation), then $\rho \sim \delta^{\prime} / 2$, $\left.u\right|_{\rho} \rightarrow 1 / E V$, and the exponential tail is over a short distance, $\zeta \sim 1 / \sqrt{\kappa}$. This is altogether different from the solution (with discontinuous $u$ ) for $\kappa=\infty$. Equation (4.33) implies that $\sigma<\rho$ only if $u_{\infty}<1 / E V \sim 1 / 16$. With $u_{\infty} \sim 0.8$, we will, in fact, have $u_{\infty}>1$ / $E V$. In this case, a solubility front does not exist, and the groundwater is undersaturated in $\mathrm{U}^{4+}$ everywhere. It follows that, in this case, $u$ and $v$ are determined from

$$
\begin{gathered}
u=u_{\infty}+\frac{1}{E V}\left(v-1-v_{\zeta}\right), \\
v_{\zeta \zeta}-v_{\zeta}=\kappa E(v-1)\left(u_{\infty}+\frac{1}{E V}\left(v-1-v_{\zeta}\right)\right),
\end{gathered}
$$

with

$$
v \rightarrow 1 \quad \text { as } \zeta \rightarrow \infty, \quad v=2 \rho / \delta^{\prime} \quad \text { on } \zeta=0,
$$


( since $\rho \ll 1$ ), and $\rho$ is determined from the continuity of $u$, which implies that

$$
2 \rho / \delta^{\prime} E V=u_{\infty}+\frac{1}{E V}\left(v-1-v_{\zeta}\right) \quad \text { on } \zeta=\rho .
$$

An explicit solution of (4.34b) is not possible, but a perturbation expansion can be developed if $\kappa \gg 1$. If we put

$$
\zeta=y / \sqrt{\kappa}, \quad v=1+\theta / \sqrt{\kappa},
$$

then, at leading order, (4.34) becomes

$$
\theta^{\prime \prime}=\theta\left(E u_{\infty}-\theta^{\prime} / V\right)
$$

with

$$
\theta \rightarrow 0 \quad \text { as } y \rightarrow \infty, \quad \theta^{\prime}(0) \sim E V u_{\infty}-1,
$$

and

$$
\rho=\frac{\delta^{\prime}}{2}(1+\theta(0) / \sqrt{\kappa})
$$

A first integral of (4.38) is

$$
E u_{\infty} V \log \left(1-\left(\theta^{\prime} / E V u_{\infty}\right)\right)+\theta^{\prime}=-\theta^{2} / 2 V,
$$

where $\theta<0$ and $\theta^{\prime}>0$. Applying the second condition of (4.39), we have

$$
\theta_{0}=-\sqrt{2 V\left(E V u_{\infty} \log \left(E V u_{\infty}\right)-E V u_{\infty}+1\right)} .
$$

The solution given by (4.41) exists precisely for $u_{\infty}>1 / E V$.

We omit discussion of the acid profile, except to note that it can be found as a quadrature.

4.2. Acid solubility dependence. Now we examine the behavior of solutions if we assume instantaneous precipitation kinetics, $\kappa=\infty$, but that

$$
f(h)=1+\alpha h,
$$

although the results of the preceding section suggest that we should be wary of the effects of the solubility equilibrium assumption.

The model equations for $u$ and $v$ are now

$$
v=f(h) \quad \text { if } u>0, \quad u=0 \quad \text { if } v<f(h),
$$

so that, if $u>0$, then

$$
-E V u_{\zeta}=\alpha\left(h_{\zeta \zeta}-h_{\zeta}\right) .
$$

We can no longer assume $u$ to be continuous. We now find that

$$
u=\frac{\alpha}{E V}\left(h-h_{\zeta}\right)+u_{\infty}
$$

for $\zeta>\rho$, while for $0<\zeta<\rho\left(-\delta^{\prime}\right)$, we have $v \sim 2 \zeta / \delta^{\prime}$ as before. The equations for $h$ and $\phi$ are thus

$$
h_{\zeta}=h_{\zeta \zeta}-\lambda \phi h, \quad-\frac{4}{15} \phi_{\zeta}=\mu \phi h,
$$


with (approximately, since $\rho \sim \delta^{\prime} \ll 1$ )

$$
\begin{aligned}
h & \rightarrow 0 \text { as } \zeta \rightarrow \pm \infty, \\
\phi & \rightarrow 1 \text { as } \zeta \rightarrow \infty, \\
{\left[h_{\zeta}\right]_{0_{-}^{0+}}^{0+}=-16 / 15 . } &
\end{aligned}
$$

A first integral, together with the boundary conditions (4.48), and the definition of $\Delta s$ via the choice that $\phi \rightarrow 1+\beta$ as $\zeta \rightarrow-\infty$, implies that

$$
\mu=\lambda \beta / 4 \text {. }
$$

Since observations suggest that $\beta \sim 4$, we have $\mu \sim \lambda$. Explicit solution of (4.47) does not seem tractable, and we satisfy ourselves with approximations for the cases of large and small $\lambda$, each of which can be found by a regular perturbation series. The first integral of (4.47) gives

$$
\phi=\phi^{ \pm}+\frac{15 \mu}{4 \lambda}\left(h-h_{\zeta}\right),
$$

where $\phi \rightarrow \phi^{ \pm}$as $\zeta \rightarrow \pm \infty$, i.e., $\phi^{+}=1, \phi^{-}=1+\beta$. Then $h$ satisfies

$$
h_{5}=h_{55}-\lambda \phi^{ \pm} h-\frac{15}{4} \mu h\left(h-h_{5}\right) \text {. }
$$

If $\lambda \sim \mu \ll 1$, then

$$
h \sim \frac{16}{15} e^{\zeta}, \quad \phi \sim \phi^{-}, \quad \zeta<0
$$

and

$$
\left.\begin{array}{c}
h \exp (-15 \mu h / 4) \sim \frac{16}{15} \exp \left(-4 \mu-\lambda \phi^{+} \zeta\right), \\
\phi \sim \phi^{+} \frac{15 \beta h}{16}\left(1+\frac{\lambda \phi^{+}}{(1-15 \mu h / 4)}\right),
\end{array}\right\} \zeta>0 ;
$$

in this case, the acid profile is relatively steep behind $z_{R}$ and decays on the longer space scale $\zeta \sim 1 / \lambda$ ahead of $z_{R}$.

On the other hand, if $\lambda \sim \mu \gg 1$, then $h, \zeta \sim 1 / \sqrt{\lambda}$, and $h$ approximately satisfies

$$
0=h_{55}-\left(\lambda \phi^{ \pm}+\frac{15}{4} \mu h_{5}\right) h
$$

of which a first integral is

$$
h_{\zeta}-\frac{4 \lambda \phi^{ \pm}}{15 \mu} \log \left|1+\frac{15 \mu}{4 \lambda \phi^{ \pm}} h_{\zeta}\right|=\frac{15}{8} \mu h^{2} .
$$

This cannot be explicitly solved, but, by a crude calculation using a Taylor series truncation of the logarithm, we obtain

$$
\begin{array}{rlrl}
h & \sim k \exp \left[\sqrt{\lambda \phi^{-}} \zeta\right], & & \zeta<0, \\
& \sim k \exp \left[-\sqrt{\lambda \phi^{+}} \zeta\right], & \zeta>0,
\end{array}
$$


with

$$
k=\frac{16}{15 \sqrt{\lambda}\left(\sqrt{\phi^{-}}+\sqrt{\phi^{+}}\right)}
$$

and

$$
\phi \sim \phi^{ \pm} \pm \frac{15}{16} \beta \sqrt{\lambda \phi^{ \pm}} h \quad \text { in } \zeta \gtrless 0 .
$$

Since nodules of dimension $1 \mathrm{~cm}$ are observed, compared with the length scale $l \sim 6$ $\mathrm{cm}$, it seems that $\lambda, \mu \gg 1$ may be the more relevant limit to consider. In any event, the $h$ and $\phi$ profiles are as shown in Fig. 4.

Finally, we compute the value of $u$ in $(0, \sigma)$. From (4.18b) and (4.46), we have, approximately,

$$
\left.E V u\right|_{\rho^{-}}=-\left.\alpha h_{\zeta}\right|_{\rho^{+}}+E V u_{\infty}+\left.\alpha h_{\zeta}\right|_{\rho^{+}}-2 \alpha / \delta^{\prime}+2 / \delta^{\prime}
$$

that is,

$$
\left.u\right|_{\rho^{-}}=u_{\infty}+2(1-\alpha) / E V \delta^{\prime} .
$$

It is evident that the aforementioned problem, wherein $u \sim 1 / \delta^{\prime}$, will occur here also, unless $\alpha \sim 1$. We therefore conclude that we cannot neglect nonequilibrium kinetics of dissolution, even if $\kappa$ is large, as the limit $\kappa \rightarrow \infty$ is not a proper singular limit of the equations. This may have important consequences for the dynamics in other, similar systems.

5. Conclusions. We have produced a one-dimensional model for the reaction-diffusion advection transport of uranium minerals through a porous host rock due to the infiltration of oxygenated groundwater. Using rapid reaction rate and solid density asymptotics [6], analytic expressions for the concentration of reactants and positions of reaction fronts have been found. In the absence of a mediating agent (uranium), it is shown that a single redox front propagates by a diffusion-controlled mechanism, as in [4]. The one-dimensional steady-state problem may be solved explicitly to determine the front velocity. Moreover, the front velocity predicted by this model also accurately predicts the velocity of the oxidation front when a mediating agent is present. This will significantly simplify the (numerical) solution of the two-dimensional fissure problem.

When the redox reaction is mediated by uranium, the front splits into two-a reduction front followed by an oxidation front. This situation is similar to that governing the propagation of roll front deposits; the spacing between our oxidation and reduction fronts is very small, however. Using the fact that the ratio of the concentrations of the infiltrating dissolved oxygen to the native pyrite is very small (i.e., $\varepsilon \ll 1$ ), we obtain analytic expressions (valid to leading order in $\varepsilon$ ) for the reaction fronts and reacting species. In particular, we show that the distance between the fronts is constant, of order $\delta^{\prime} \ll 1$. This reflects the fact that the overall redox reaction is mediated by uranium, and, since the ratio of mobile (aqueous) $\mathrm{U}^{4+}$ to oxygen (or oxygen equivalent) (i.e., $\delta^{\prime}$ ) is very small, the reaction fronts remain very close to each other over the timescales of relevance. We find that, if the solubility characteristics of $\mathrm{U}^{4+}$ are treated as if in equilibrium, then unrealistic results are obtained, while the inclusion of nonequilibrium kinetics allows a more practical answer to be obtained.

The process we have established is not specific to uranium-mediated redox reactions. It describes any infiltration reaction situation in which the reaction rates are large and mediated by a secondary species. For larger values of $\delta^{\prime}$, we have described here a mech- 
anism for concentrating (ore) deposits in extended veins. A question of interest is to see whether such split-front solutions are morphologically stable.

Acknowledgments. The authors thank Intera Environmental Consultants, Ltd., Dr. W. T. Shaw, and Prof. I. Neretnieks for bringing this problem to our attention and for many useful discussions. We also wish to thank Dr. P. Grindrod and Dr. S. M. Sharland for helpful discussions. We also thank Dr. S. M. Sharland for providing Fig. 1.

\section{REFERENCES}

[1] J. E. Cross A. Haworth, I. Neretnieks, S. M. Sharland, and C. J. Tweed, Modelling of redox front and uranium movement in a uranium mine at Poços de Caldas, in Proc. 2nd Internat. Conf. on Chemistry and Migration Behaviour of Actinides and Fission Products in the Geosphere, Monterey, CA, Nov. 6-10, 1989; Radiochim. Acta, 1990, to appear.

[2] Y. HeKIM AND H. S. FogLER, On the movement of multiple reaction zones in porous media, AIChE J., 26 (1980), pp. 403-411.

[3] P. C. LICHTNER, The quasi-stationary state approximation to coupled mass transport and fluid-rock interaction in a porous medium, Geochim. Cosmochim. Acta, 52 (1988), pp. 143-165.

[4] P. Ortoleva, G. Auchmuty, J. Chadam, J. Hettmer, E. Merino, C. H. Moore, and E. Ripley, Redox front propagation and banding modalities, Phys. D, 19 (1986), pp. 334-354.

[5] P. Ortoleva J. Chadam, E. Merino, And A. Sen, Geochemical self-organization II: The reactiveinfiltration instability, Amer. J. Sci., 287 (1987), pp. 1008-1040.

[6] P. Ortoleva, E. Merino, C. MORSE, AND J. ChADAM, Geochemical self-organisation I: Reaction-transport feedbacks and modeling approach, Amer. J. Sci., 287 (1987), pp. 979-1007.

[7] G. O. RoBerts, Fast Benard convection, Geophys. Astrophys. Fluid Dynamics, 12 ( 1979), pp. $235-272$.

[8] J. Smellie, N. Chapman, I. MCKinley, E. P. Franca, And M. Shea, Testing safety assessment models using natural analogues in high natural-series groundwaters. The second year of the Poços de Caldas project, Mat. Res. Soc. Sympos. Proc., 127 (1989), pp. 863-870.

[9] R. Sultan, P. Ortoleva, F. De Pasquale, and P. TaRTaglia, Bifurcation of the Ostwald-Liesegang supersaturation-nucleation-depletion cycle, Earth Sci. Rev., 29 (1990), pp. 163-173.

[10] M. P. WALSh, S. L. BRYANT, R. S. SCHECHTER, AND L. W. LAKE, Precipitation and dissolution of solids attending flow through porous media, AIChE J., 30 (1984), pp. 317-318. 